\title{
MicroRNAs in cancer translational research: the microcosm of cancer diagnosis, prognosis, and therapy
}

\section{Dominic Rose*}

Bioinformatics Group, Department of Computer Science, University of Freiburg, Freiburg, Germany

*Correspondence: dominic@informatik.uni-freiburg.de

\author{
A book review on \\ MicroRNAs in cancer translational research \\ by William C. S. Cho, Springer, 1st Edn, 2011, XIII, 557 pages. ISBN: 978-94-007-0297-4
}

The discovery of microRNAs (miRNAs) almost 20 years ago rapidly changed our perception of eukaryotic gene regulation. MiRNAs apparently function as post-transcriptional regulators mediating translational repression, target degradation, and gene-silencing. They furthermore have been recognized to coordinate cellular proliferation, differentiation, apoptosis, and development. In fact, this prominent and currently most widely studied class of small non-coding RNAs has been linked to manifold molecular mechanisms, including a plethora of physiological processes, but also to disease pathophysiology. Aberrant miRNA levels are observed for multiple human diseases. Beyond their emerging roles in inherited, neurological, or cardiovascular diseases, miRNA-controlled gene expression is an intriguing phenomenon observed in virtually all cancer types. With the observation that miRNA expression profiles of human tumors show intrinsic defects in miRNA production, miRNAs became key molecules in translational research and oncology. They bear a great potential as diagnostic markers and therapeutic targets and are considered to subsequently open the road for a wide spectrum of revolutionary clinical applications. In particular, miRNAs are expected to reliably mark differentiation states and to serve as both oncogenes and tumor suppressors. Current therapies targeting miRNA overexpression include miRNA sequestration approaches that rely on complementaritybased miRNA inhibition, i.e., by the use of synthetic anti-miRNAs or miRNA sponges. Downregulated miRNA levels, which are globally observed in tumor tissue, can be encountered by epigenetic drug treatment in order to re-establish normal miRNA expression. They can be tackled by miRNA delivery systems generating the missing miRNAs or by drugs targeting the miRNA biogenesis pathway, therefore enhancing miRNA processing and production.

In his book "MicroRNAs in cancer translational research" (MCTR) the editor William C. S. Cho provides a comprehensive coverage and a succinct overview of the state-of-the-art knowledge on miRNA research (Cho, 2011). The book represents the signs of the times with respect to cancer diagnosis, prognosis, and therapy. On more than 550 pages, structured into 22 chapters, MCTR brings together leading researchers in the field reviewing recent findings and cutting-edge discoveries targeted toward cancer treatment.

MiRNA research is a story of success. As tellingly stated in the preface by the editor, miRNAs caused a paradigm shift in cancer translational research and oncology. Today, there are already pioneering clinical trials on the way to clarify and potentially establish miRNAs in disease treatment. With the advent of high-throughput techniques and next generation sequencing just a decade ago, novel seminal, and exciting miRNArelated findings were published in quick succession. In fact, the number of publications on miRNAs increased exponentially in recent years. As a consequence, it is almost impossible to follow the rush of miRNAliterature in detail. Therefore, it is sound and timely to collect and converge the various results of current miRNA research in a single comprehensive book.

MCTR starts with a general introduction on miRNAs, fundamental mechanisms causing their dysregulation in cancer and their roles as cancer classifiers, as prognosis and drug response predictors, and as cancer biomarkers. The book then catches the readers interest with 14 well-structured chapters on specific cancer types and associated miRNAs. A novel way to structure a book on miRNAs which clearly makes it unique. When describing the various subtypes, MCTR aptly begins with an in-depth analysis of miRNAs and their role in lung cancer, which is the most frequent type of cancer and the leading cause of cancer death worldwide. Subsequently, miRNA-related research highlights and future perspectives on cancer stem cells, invasion, metastasis, radio- and chemotherapy response, anticancer drug resistance, cancer metabolism, and blood-based biomarkers are compiled. The book closes with an accurate and compelling overview on RNAi-based treatment of brain tumors. Finally, an index of alphabetically sorted terms, together with their respective page-number they occurred, is given. A glossary of index terms is missing but would be exceedingly useful.

MCTR uses a combination of comprehensive tables, figures, and graphs to relay its essentials. All chapters are elegantly reviewed and highly citable. Each starts with a sufficient introduction, for example of the particular cancer subtype. This allows to read them independent from each other, a feature that readers will highly acknowledge. In turn, a few chapters would have been benefited from a more rigorous editing. For example, it is not necessary to repeatedly introduce miRNAs as "novel class of small non-coding RNAs that are 22 nucleotides in length" or as "small regulators with roles in cell proliferation, differentiation, and apoptosis." Throughout all chapters, numerous examples of specific differentially expressed miRNAs that have been identified to play crucial key regulatory 
roles are provided. These lists are of utmost interest but obviously too long. For example, Table 1.3 comprises more than 25 pages. Such excessive tables should be part of an appendix, they can be distracting and the danger to loose the readers attention is imminent. Nonetheless, this putative caveat in no way detracts from the overall quality of the chapters. Another minor criticism might be that some figures are of poor quality and could have been provided with better solutions.

MCTR is not a classical text book. It can be seen as the encyclopedia in the field, a rich and thorough compendium uniting all relevant aspects of miRNAstimulated translational cancer research. It covers, however, a proper spectrum of relevant topics and thus can be recommended for a broad scientific and medical readership. MCTR is a precious book for all types of readers: cancer researchers, oncologists, pathologists, biologists, clinical chemists, pharmacologists, and even bioinformaticians.

In summary, its not only miRNAs that bear a great potential. MCTR can serve as the reference text book in miRNA-based cancer translational research stimulating the scientific community.

\section{REFERENCE}

Cho, W. (2011). MicroRNAs in Cancer Translational Research. Heidelberg: Springer.

Received: 22 February 2012; accepted: 03 March 2012; published online: 21 March 2012.

Citation: Rose D (2012) MicroRNAs in cancer translational research: the microcosm of cancer diagnosis, prognosis, and therapy. Front. Gene. 2:42. doi: 10.3389/ fgene.2012.00042

This article was submitted to Frontiers in Non-Coding RNA, a specialty of Frontiers in Genetics.

Copyright $\odot 2012$ Rose. This is an open-access article distributed under the terms of the Creative Commons Attribution Non Commercial License, which permits non-commercial use, distribution, and reproduction in other forums, provided the original authors and source are credited. 